\title{
3D Evaluation of Distalization of Maxillary First Permanent Molar Using Mini Implants: A Finite Element Study
}

\section{Rajivi Kalpakuri ${ }^{1}$, Manish Pisarla ${ }^{2}$, Tejaswi Kala ${ }^{3 *}$, Jadhav Sachin Kumar $^{4}$, B Jaya Lakshmi ${ }^{5}$ and B Prashanth Kumar ${ }^{6}$}

${ }^{1}$ Assistant Professor, Department of Orthodontics and Dentofacial Orthopaedics, Vydehi Institute of Dental Sciences, Bangalore, Karnataka, India

${ }^{2}$ Assistant Professor, Department of Orthodontics and Dentofacial Orthopaedics,

Meghana Institute of Dental Sciences, Nizamabad, Telangana, India

${ }^{3}$ Assistant Professor, Department of Public Health Dentistry, Tirumala Institute of

Dental Sciences, Nizamabad, India

${ }^{4}$ Post Graduate Student, Department of Public Health Dentistry, Post Graduate

Institute of Dental Sciences, Rohtak, Haryana, India

${ }^{5}$ Assistant Professor, Oral Medicine and Radiology, Tirumala Institute of Dental

Sciences, Nizamabad, India

${ }^{6}$ Assistant Professor, Department of Pedodontics and Preventive Dentistry, Tirumala

Institute of Dental Sciences, Nizamabad, India

*Corresponding Author: Tejaswi Kala, Assistant Professor, Department of Public

Health Dentistry, Tirumala Institute of Dental Sciences, Nizamabad, India.
Received: July 06, 2021

Published: September 14, 2021

(C) All rights are reserved by Tejaswi Kala., et al.

\section{Abstract}

Objectives: The purpose of the study was to use FEM to analyze three dimensional movements of first permanent molar distalization using mini-implants.

Aim of the Study: The aim of the study was to analyze the FEM model of the maxilla which simulates the three dimensional movements of the first permanent molar after distalization using mini-implants.

Materials and Methods: In the present study, a 3D FEM model of right maxilla with an end on molar relation was created and was used to calculate the amount of distalization in three axes, when forces were applied on implant to distalize the molar.

Results: On buccal side, the molar showed mesial buccal rotation, intrusion with uncontrolled distal tipping on application of 150 gms, on application of 200 gms, the molar showed mesial buccal rotation, intrusion with uncontrolled distal tipping and extrusion of mesial cusps. On palatal side, the molar showed mesio palatal rotation, distal displacement, and intrusion of distal cusps and extrusion of mesial cusps which were the same on application of 150 and 200 gms of force, but the movements were more with the latter. Interpretation and Conclusion: Buccal placement leads to distalization and unwanted tipping, whereas palatal placement shows more distalization and less tipping, as it is closer to center of resistance. In terms of visibility and ease of placement, buccal implant was easier compared to palatal implant.

Keywords: Mini-Implant; Distalization; Skeletal Anchorage 


\section{Introduction}

Maxillary molar distalization is a non-extraction treatment option for maxillary arch expansion. The rationale for maxillary molar distalization is a Class II relationship (as a result of maxillary posterior teeth migrating mesially) or a small skeletal Class II relationship with minimal skeletal deformity or no mandibular tooth size-arch length discrepancy [1].

The traditional method used for maxillary molar distalization is headgear (extra-oral anchorage). However, headgear has some disadvantages; it is esthetically unacceptable and needs patient compliance [2]. These disadvantages led to the development of intraoral distalization methods that do not require patient cooperation: e.g. magnets [3], nickel-titanium open-coil springs [4], pendulum appliance [5], Keles slider appliance [6], distal jet appliance [7] and several other methods. The common and unwanted side effect of these non-compliance methods is the mesial drift of the premolars and the incisors: i.e. anchorage loss [8].

To prevent anchorage loss, intraoral distalization methods take support from the skeletal structures with the help of temporary anchorage devices such as endosseous implants, mini plates and mini screws [9]. Implants and mini plates have some disadvantages such as high costs and the need for additional surgery for placement and removal. Mini-implants, on the other hand, are preferred because they are cheaper and less invasive than other methods [10]. Additionally, they can be easily placed in several skeletal regions. The anterior palate is considered to be a safe region for mini-implant insertion because it is far from the tooth roots and important anatomic structures [11,12]. Another benefit of palatal anchored distalization mechanics would be that no fixed appliances are required during the distalization period.

Many authors have examined the effects of intraoral distalization performed with support from the mini-implants placed in this region. However, the tooth movements were generally analysed two dimensionally on cephalometric radiographs and dental cast photocopies in these studies.

The FE approach was proposed into dental biomechanical research in 1973 (Farah., et al.) and has been widely used to investigate the stress and strain fields in alveolar support structures ever since. The FEM is a powerful tool for the analysis of complex structures [8]. It has the advantages that it could be done in silico and is reliable for numerical approximation to all physical problems that can be modelled by a differential equation description.

The Finite Element Method (FEM) is a numerical method of analysis that allows the study of stress distribution in biological systems. In dentistry, it has been used in studies of cranio-facial development, prosthetics and implantology.

This method enables the strain-stress in the interior of the structures to be calculated. It also has the potential for equivalent mathematical modelling of a real object of complicated three dimensional geometry. At the same time, it permits the application of various force systems at a set point and the study of the distribution of such forces through the structures like alveolar bone, tooth and periodontal ligament.

\section{Aim of the Study}

The aim of the study was to use FEM to analyze three dimensional movements of first permanent molar distalization using mini-implants.

\section{Materials and Methods}

The present study was conducted for 3D evaluation of distalization of maxillary first permanent molar using mini-implants by using a Finite Element Method.

\section{Armamentarium}

1. Workstation computer with following configuration:

a. Intel core 2 duo with $2.1 \mathrm{GHz}$

b. 2 GB of RAM

c. $\quad 2 \mathrm{~GB}$ graphics card

d. 320GB hard disc

e. 17 " monitor.

2. Spiral C.T scan machine: An X-force/SH spiral C.T scan machine was used for taking the CT scan images of the skull (from which only region of interest like maxilla was extracted).

3. Software's used: 
a. Mimics 8.11: Materialise's interactive medical image control system (MIMICS)

b. ANSYS 12.1: Analysis system software

c. Hypermesh 13.0 .

Selection of CT images: The sample for the present study included non-pathological CT images of intact maxilla. The CT images for the required study were retrospectively collected. The CT scans of the patient were imaged using a X-force/SH spiral C.T scan machine. The scans for these patients were ordered by other clinicians for specific clinical indications like sinusitis. The collected images were evaluated according to the stipulated criteria.

The inclusion criteria for the selection of CT images were:

\section{General criteria}

- $\quad$ Non pathological maxilla

- No metallic restorations and other artefacts

- No periodontal abnormalities

- $\quad$ Non ankylosed teeth

- $\quad$ Vital teeth

- Skeletal class I jaw relation

- $\quad$ Anterior crowding.

- $\quad$ End on molar relation.

Data acquisition and model generation

- $\quad$ The CT Scan data is processed using Mimics 8.11 software and extracted the maxilla portion for the study which is further taken into Rapid form 2014 Software and converted into geometric model.

- The geometric model is further imported into Hypermesh 13.0 software to convert into finite element model.

- $\quad$ Bones, teeth, PDL, are separated and also physical metal parts like brackets, wire and implants are modeled using reverse engineering technique.

- Two separate models were created for the study:

- Implants placed on palatal side.
- Implants placed on buccal side.

- Material properties are assigned to each part (Elastic modulus and poisson ratio), loads and boundary conditions were applied and then the models are exported to simulation software.

- $\quad$ ANSYS 12.1 was used for analysis and was carried out for each models separately subjected to 150 gms and 200 gms of loads.

- The results like movement of first molar were derived.

\section{Observations and Results}

Buccal side

Initially, the stability of maxillary buccal implants was questioned. With the development of new implants, there is no reason to refrain from use of buccal implants in patients below 15 years and older. The use of buccal implants is preferable because of their ease of implantation and simple application in treatment.

Buccal interdental implants may have the disadvantage of impeding adjacent tooth movement. If properly positioned, however with 2 to $3 \mathrm{~mm}$ of distal movement on either side, there is no little possibility that tooth movement will be restricted, to be precise, buccal alveolar bone is being used rather than the interdental alveolar bone. If buccal space is used with accurate implant positioning, movement of at least a half cusp width of molar is possible.

In the present study, mini-implant with a $1.2 \mathrm{~mm} \times 7 \mathrm{~mm}$ diameter were placed buccally at an angle of 90 degrees in between second premolar and first molar. As the molar distalizing force was applied at the level of the bracket, on application of $150 \mathrm{gms}$, the first molar showed:

- $\quad-0.001 \mathrm{~mm}$ mesial buccal rotation (Figure 1).

- $\quad 0.002 \mathrm{~mm}$ uncontrolled distal tipping (Figure 2).

- $\quad 0.001 \mathrm{~mm}$ intrusion (Figure 3).

As the molar distalizing force was applied at the level of the bracket, on application of $150 \mathrm{gms}$, the second molar showed:

- $\quad$ No rotation (Figure 4).

- $\quad 0.002 \mathrm{~mm}$ distal tipping (Figure 5).

- $\quad$ Intrusion (Figure 6). 
In the present study, mini-implant with a $1.2 \mathrm{~mm} \times 7 \mathrm{~mm}$ diameter were placed buccally at an angle of 90 degrees in between second premolar and first molar. On application of $200 \mathrm{gms}$, the first molar showed:

- $\quad 0.001 \mathrm{~mm}$ mesial buccal rotation (Figure 1).

- $\quad 0.003 \mathrm{~mm}$ uncontrolled distal tipping (Figure 2).

- $\quad 0.001 \mathrm{~mm}$ intrusion and extrusion of mesial cusps (Figure $3)$.

On application of 200 gms, second molar showed:

- $\quad-0.001 \mathrm{~mm}$ mesial buccal rotation (Figure 4).

- $\quad 0.002 \mathrm{~mm}$ uncontrolled distal tipping (Figure 5).

- $\quad 0.001 \mathrm{~mm}$ intrusion (Figure 6).

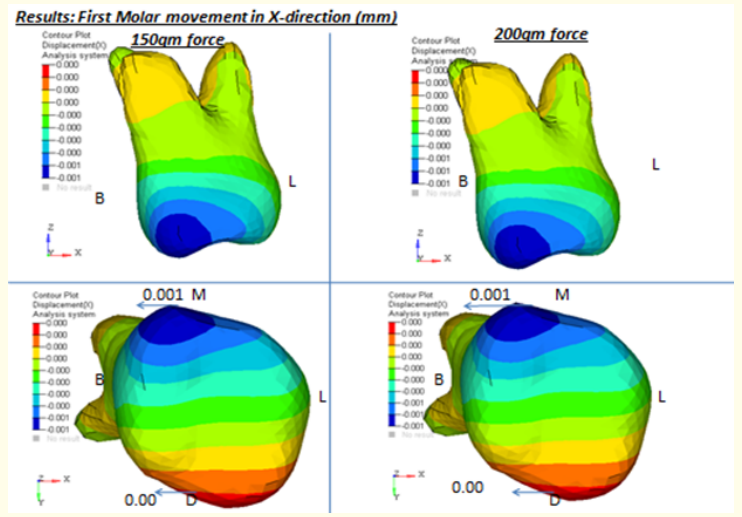

Figure 1: First molar movement in X-direction (mm).

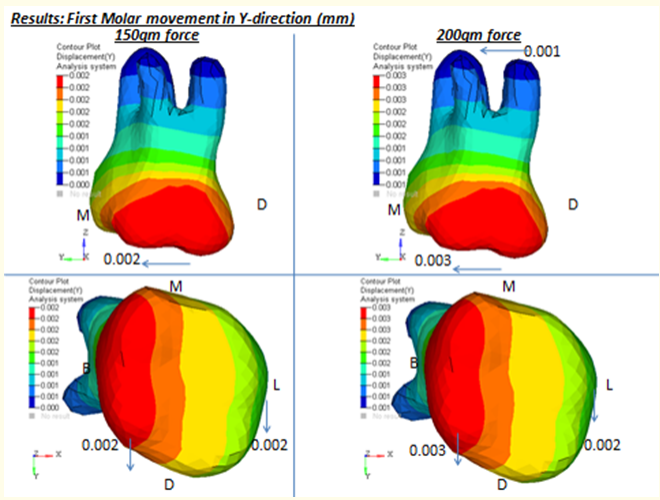

Figure 2: First molar movement in Y-direction (mm).

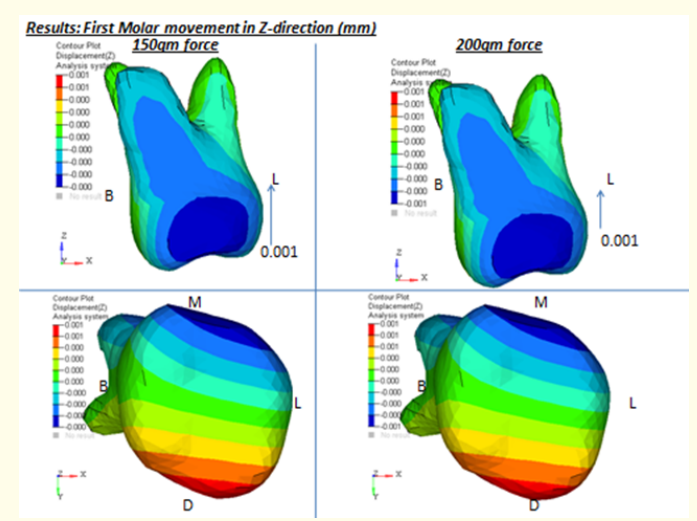

Figure 3: First molar movement in Z-direction (mm).

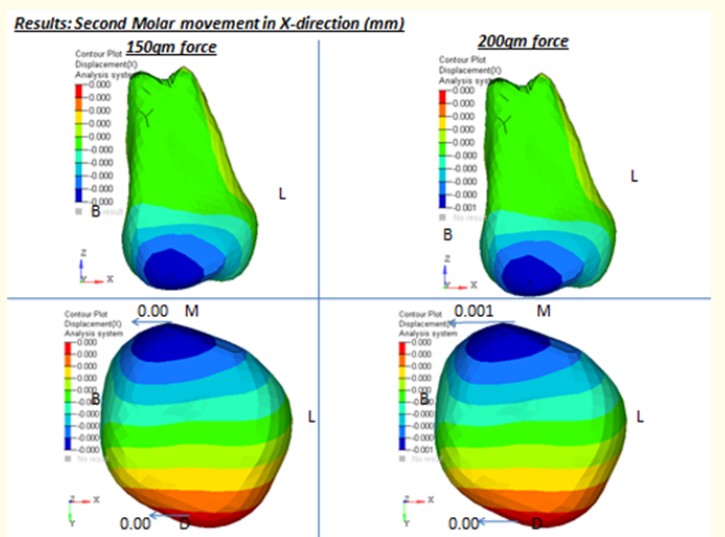

Figure 4: Second molar movement in X-direction (mm).

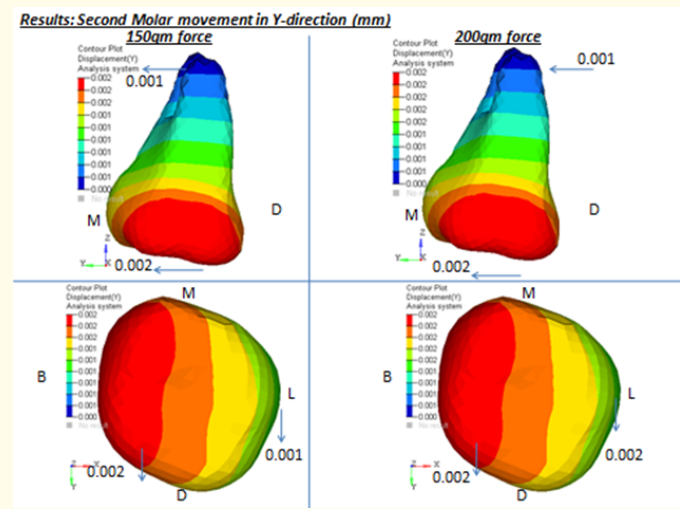

Figure 5: Second molar movement in Y-direction (mm). 


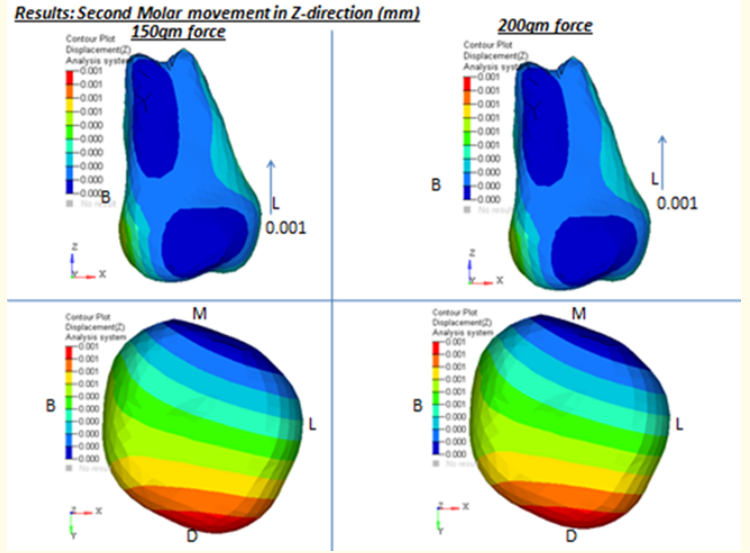

Figure 6: Second molar movement in Z-direction (mm).

Palatal side

Placing the mini-implants between the buccal roots, limits the distal movement of the dentition (Lee., et al. 2009). Additionally, this movement results in tilting, most notably in the molars.

To achieve molar distalization in MIA-TPA, direct traction using elastic modules stretching from a mini-implant to a TPA helix is suggested. Mini-implant were inserted into the palate in this study, where in attached gingiva is not a major concern, in contrast to the buccal side.

The mesio distal axis of molar movement can be controlled by adjusting the vertical position of mini-implants and/or the direction of the line of action relative to furcation. Thus, rather than tipping, bodily tooth movement is encouraged.

The MIA-TPA is a dental appliance that can successfully establish absolute anchoring and distalize maxillary first molars. This movement is inextricably linked to expansion. Extrusion is hindered by the apical distal force, and molar movement is slow relatively.

In this study, when mini-implant assisted trans palatal arch was used for molar distalization, on application of $150 \mathrm{gms}$, first molar showed:

- $\quad 0.001 \mathrm{~mm}$ mesio palatal rotation (Figure 7).

- $\quad 0.002 \mathrm{~mm}$ distal displacement (Figure 8).

- $\quad 0.001 \mathrm{~mm}$ intrusion of distal cusps (Figure 9).
- $\quad 0.001 \mathrm{~mm}$ extrusion of mesial cusps (Figure 9).

In this study, when mini-implant assisted trans palatal arch was used for molar distalization, on application of $150 \mathrm{gms}$, second molar showed:

- $\quad$ No rotation (Figure 10).

- $\quad 0.002 \mathrm{~mm}$ distal displacement (Figure 11).

- $\quad 0.001 \mathrm{~mm}$ intrusion (Figure 12).

In this study, when mini-implant assisted trans palatal arch was used for molar distalization, on application of 200 gms, the first molar showed:

- $\quad 0.002 \mathrm{~mm}$ mesio palatal rotation (Figure 7).

- $\quad 0.004 \mathrm{~mm}$ distal displacement (Figure 8).

- $\quad 0.001 \mathrm{~mm}$ intrusion of distal cusps (Figure 9).

- $\quad 0.001 \mathrm{~mm}$ extrusion of mesial cusps (Figure 9).

In this study, when mini-implant assisted trans palatal arch was used for molar distalization, on application of $200 \mathrm{gms}$, second molar showed:

- $\quad-0.001 \mathrm{~mm}$ mesio palatal rotation (Figure 10).

- $\quad 0.003 \mathrm{~mm}$ distal displacement (Figure 11).

- $\quad 0.001 \mathrm{~mm}$ intrusion (Figure 12).

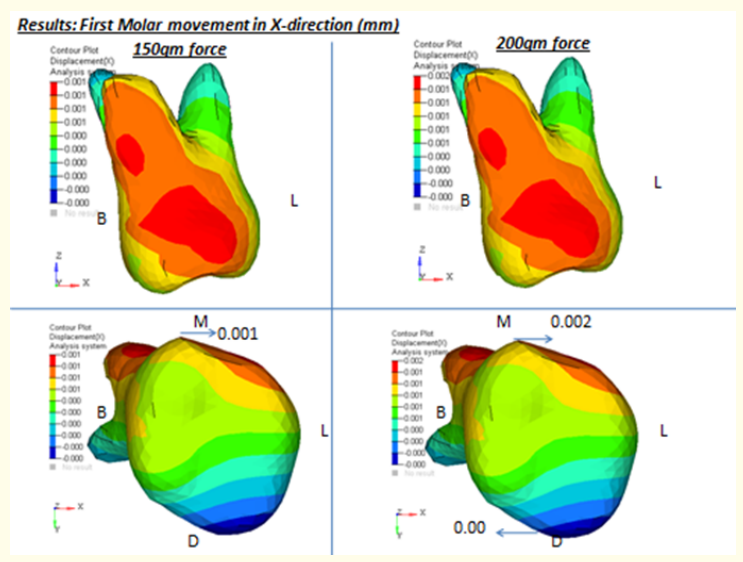

Figure 7: First molar movement in X-direction (mm). 


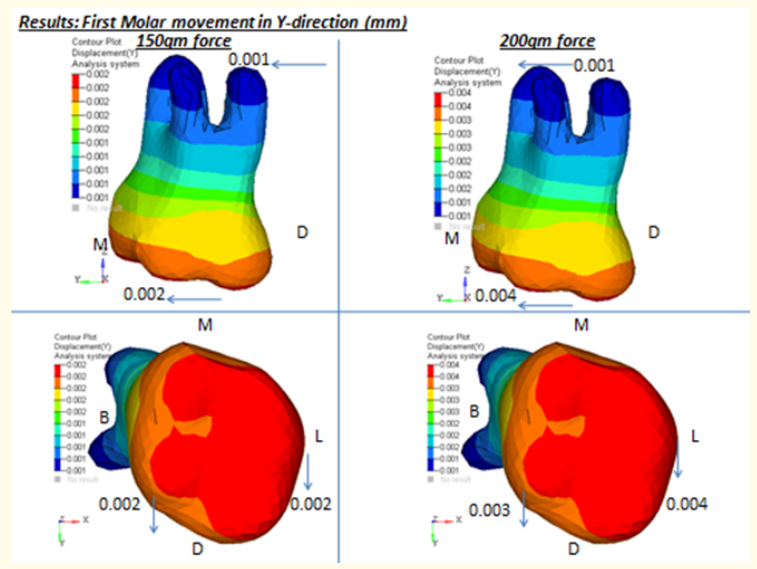

Figure 8: First molar movement in Y-direction (mm).

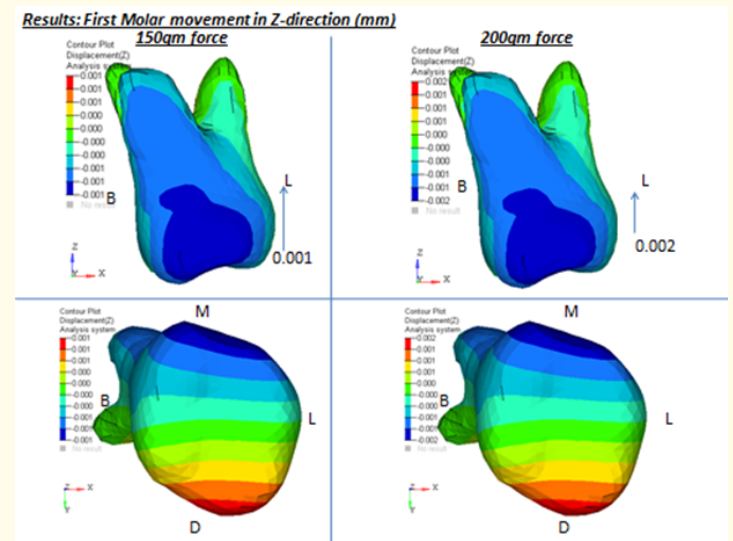

Figure 9: First molar movement in Z-direction (mm).

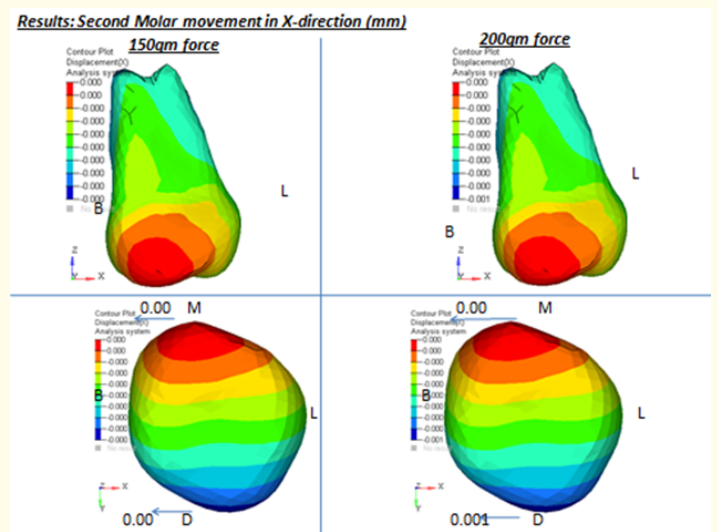

Figure 10: Second molar movement in X-direction (mm).

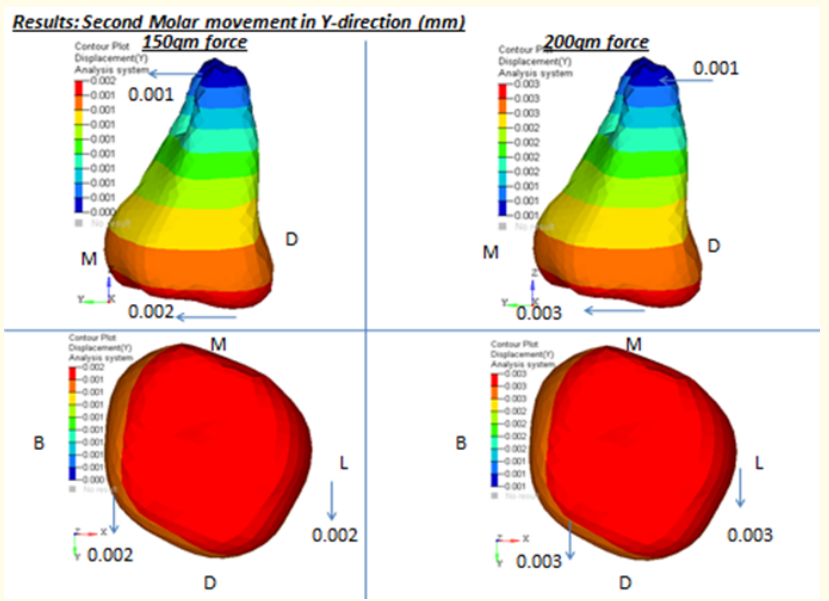

Figure 11: Second molar movement in Y-direction (mm).

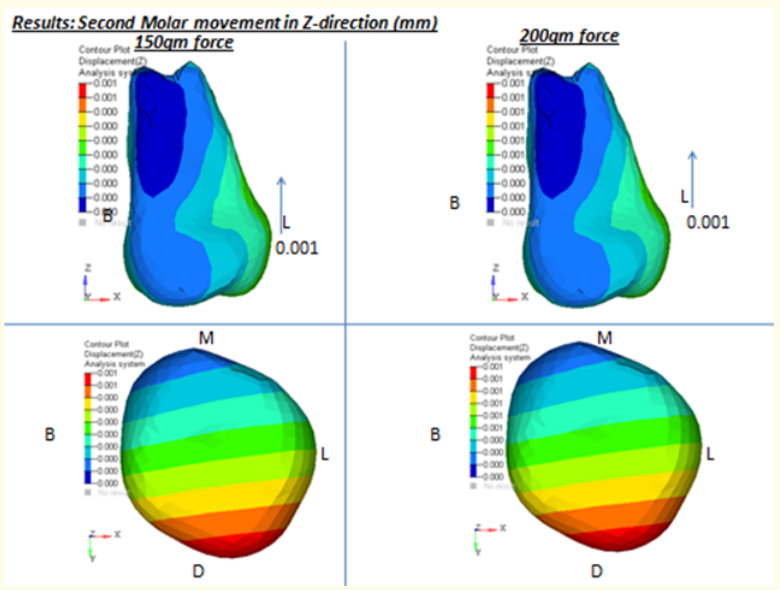

Figure 12: Second molar movement in Z-direction (mm).

Buccal side

As the molar distalizing force was applied at the level of the bracket, on application of 150 gms, the first molar showed, -0.001 $\mathrm{mm}$ mesial buccal rotation, $0.002 \mathrm{~mm}$ uncontrolled distal tipping, $0.001 \mathrm{~mm}$ intrusion. The second molar showed, no rotation, 0.002 $\mathrm{mm}$ distal tipping, intrusion (Table 1).

On application of $200 \mathrm{gms}$, the first molar showed, $-0.001 \mathrm{~mm}$ mesial buccal rotation, $0.003 \mathrm{~mm}$ uncontrolled distal tipping, 0.001 mm intrusion and extrusion of mesial cusps. The second molar showed, $-0.001 \mathrm{~mm}$ mesial buccal rotation, $0.002 \mathrm{~mm}$ uncontrolled distal tipping, $0.001 \mathrm{~mm}$ intrusion (Table 1 ). 


\section{Palatal side}

In this study, when mini-implant assisted trans palatal arch was used for molar distalization, on application of 150 gms, first molar showed, $0.001 \mathrm{~mm}$ mesio palatal rotation, $0.002 \mathrm{~mm}$ distal displacement, $0.001 \mathrm{~mm}$ intrusion of distal cusps, $0.001 \mathrm{~mm}$ extrusion of mesial cusps. The second molar showed, no rotation, $0.002 \mathrm{~mm}$ distal displacement, $0.001 \mathrm{~mm}$ intrusion (Table 1).

On application of $200 \mathrm{gms}$, the first molar showed, $0.002 \mathrm{~mm}$ mesio palatal rotation, $0.004 \mathrm{~mm}$ distal displacement, $0.001 \mathrm{~mm}$ intrusion of distal cusps, $0.001 \mathrm{~mm}$ extrusion of mesial cusps. The second molar showed, -0.001 mesio palatal rotation, $0.003 \mathrm{~mm}$ distal displacement, $0.001 \mathrm{~mm}$ intrusion (Table 1).

\begin{tabular}{|c|c|c|c|c|c|c|c|}
\hline \multirow{2}{*}{ Tooth } & \multirow{2}{*}{ Side } & \multicolumn{3}{|c|}{$\mathbf{1 5 0}$ gms } & \multicolumn{3}{c|}{ 200 gms } \\
\cline { 3 - 8 } & & $\mathbf{X}$ & $\mathbf{Y}$ & $\mathbf{Z}$ & $\mathbf{X}$ & $\mathbf{Y}$ & $\mathbf{Z}$ \\
\hline First molar & Buccal & -0.001 & 0.002 & 0.001 & -0.001 & 0.003 & 0.001 \\
\cline { 3 - 8 } $\begin{array}{c}\text { Second } \\
\text { molar }\end{array}$ & side & 0.000 & 0.002 & 0.001 & -0.001 & 0.002 & 0.001 \\
\hline $\begin{array}{c}\text { First molar } \\
\text { Palatal }\end{array}$ & 0.001 & 0.002 & 0.001 & 0.002 & 0.004 & 0.002 \\
\cline { 1 - 6 } $\begin{array}{c}\text { Second } \\
\text { molar }\end{array}$ & side & 0.000 & 0.002 & 0.001 & -0.001 & 0.003 & 0.001 \\
\hline
\end{tabular}

Table 1: Displacement summary table for both buccal and palatal side.

On application of different loads i.e. 150 gms and 200 gms on first and second molar:

- The distal movement on buccal side varied as the load increased from 150 gms to 200 gms, first molar moved from $0.002 \mathrm{~mm}$ to $0.003 \mathrm{~mm}$, second molar movement was constant (Graph 1).

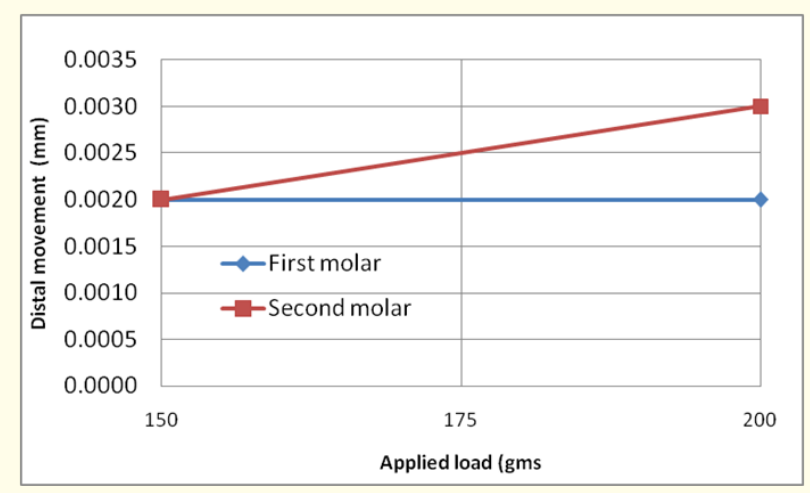

Graph 1: Buccal side distal movement variation with applied load.
- The distal movement on palatal side varied as the load increased from 150 gms to 200 gms, first molar moved from $0.002 \mathrm{~mm}$ to $0.004 \mathrm{~mm}$ and second molar moved from 0.002 $\mathrm{mm}$ to $0.003 \mathrm{~mm}$ (Graph 2).

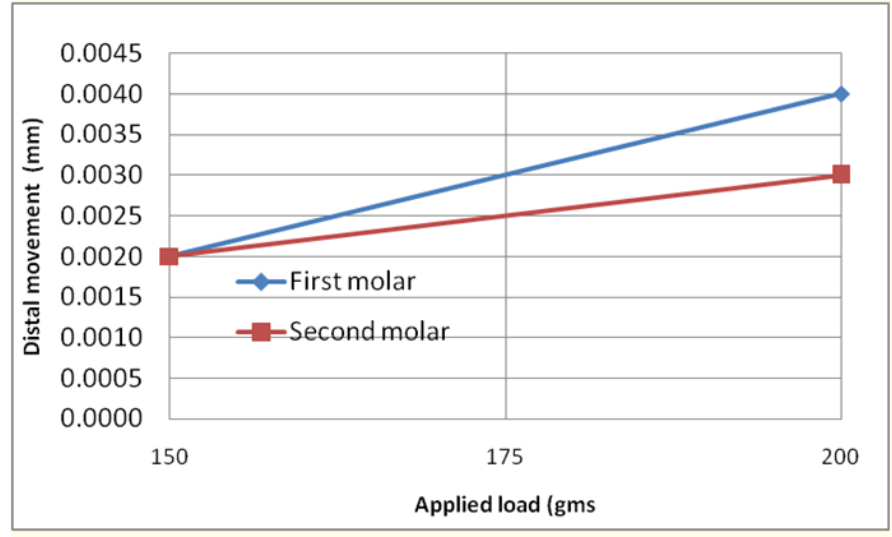

Graph 2: Palatal side distal movement variation with applied load.

- On application of 150 gms, distal movement of first molar when mini-implant was placed either buccal or palatal is the same. On application of 200 gms, distal movement of first molar when mini-implant was placed palatally is more than buccal placement (Graph 3).

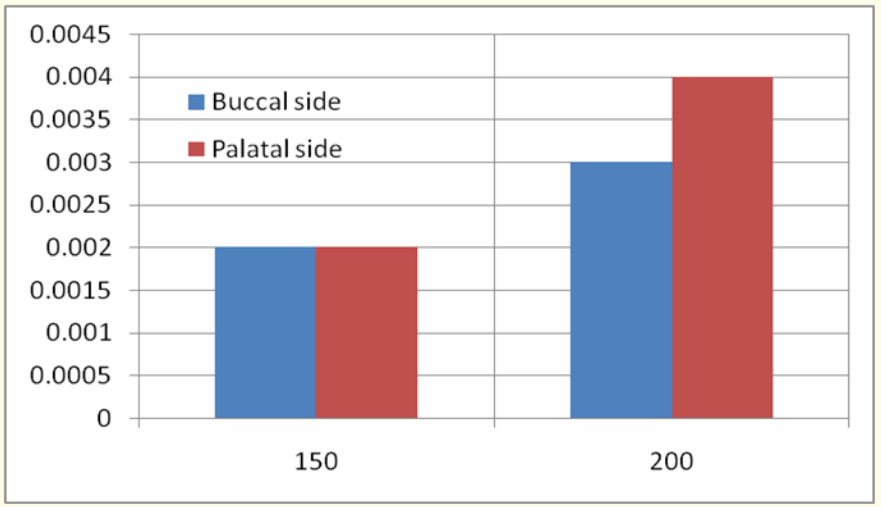

Graph 3: First molar distal movement comparison. 
- $\quad$ On application of 150 gms, distal movement of second molar when mini-implant was placed either buccal or palatal is the same. On application of 200 gms, distal movement of second molar when mini-implant was placed palatally is more than buccal placement (Graph 4).

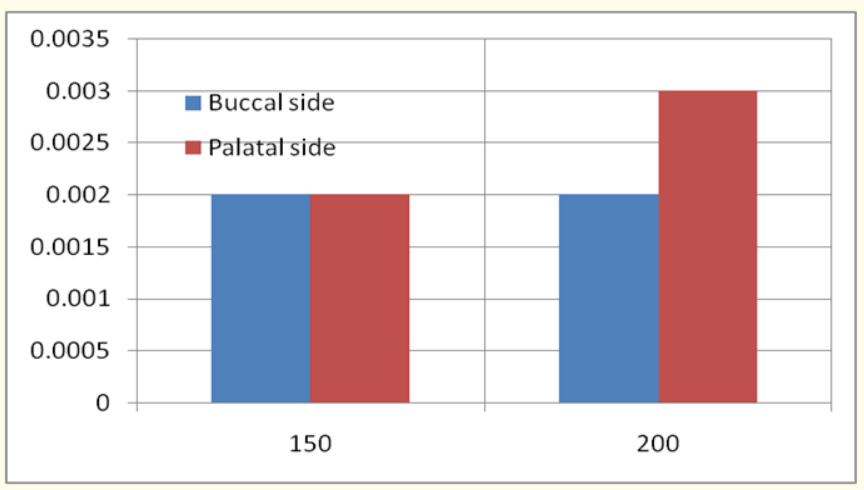

Graph 4: Second molar distal movement comparison.

\section{Discussion}

Anchorage management has historically been a tough and unpredictable task in orthodontic practise. Numerous attempts have been made to obtain bone tissue support for successful anchoring management in order to overcome this obstacle. In the early stages of orthodontic research, several case reports described osseo integrated implants for restoration of missing teeth and later for anchorage in orthodontic treatment. This led to the modification of prosthodontic dental implants and the development of a new implant system for orthodontic anchorage. These skeletal anchorage systems have been subsequently used as onplants, osseo integrated implants, zygomatic ligatures, mini plates and more recently miniimplants. Mini-implants have the advantages of low cost, simple surgical placement and ease of removal. They are small enough to be placed in any space in the alveolar bone, even in the interdental areas. The use of mini- implant has now been expanded, but there are still many unknown factors that could affect the clinical success of mini-implants.

\section{About distalization}

Headgear has been a conventional modality for Class II malocclusion through distalization of molars or entire the maxillary dentition. However, its primary disadvantage is its reliance on patient cooperation, which can be challenging given social and aesthetic issues. To avoid this drawback, various non-compliance appliances have been developed including the Keles slider, repelling magnets, distal jet and pendulum. These devices distalized maxillary molars continuously, which may result in distal tipping and extrusion of the first molars, whilst mesial reactive forces may result in anchoring loss and labial flaring of anterior teeth. To overcome these unwanted side- effects, skeletal anchorage systems have been applied in previous studies for molar distalization [57].

Orthodontists regularly encounter mild to moderate Class II molar and canine relationships that they desire to avoid treating with premolar extraction and for which supplemental treatment is frequently ineffective due to low patient compliance. Many studies on intraoral maxillary molar distalization without patient cooperation have been performed, in order to solve the patient compliance problem associated with extraoral distalization appliances [44]. Molar distalization is recommended for correction of Class II malocclusion or maxillary crowding cases without extraction [57].

It has been reported that the mean distalization possible in the sagittal direction was $3.34 \mathrm{~mm}$ with tooth-borne intraoral distalization methods and $5.10 \mathrm{~mm}$ with skeletal anchorage [45]. The distal jet moved the crowns of maxillary molars with $2.8 \mathrm{~mm}$ distally in 2.5 months. The conventional intra oral appliances distalized maxillary first molar at the rate of 0.6 to $1.2 \mathrm{~mm}$ per month.

The following observations were made in the study.

\section{Buccal side}

Initially, the stability of maxillary buccal implants was questioned. With the development of new implants, there was no reason to refrain from use of buccal implants in patients below 15 years and older. The use of buccal implants is preferable because of their ease of implantation and simple application in treatment.

Buccal interdental implants have the potential limitation of hindering the movement of adjacent teeth. If properly positioned, however with 2 to $3 \mathrm{~mm}$ of distal movement on either side, there is no possibility that tooth movement will be restricted. To be precise, buccal alveolar bone is being used rather than the interdental alveolar bone.

In this regard, mini-implants are considered as viable option due to the various advantages which include: 
- Minimum anatomic limitation in its placement.

- $\quad$ Easy placement and removal.

- $\quad$ Economical, no necessity for complicated clinical and laboratory phases, or for osseo integration when mini-implants are used.

- $\quad$ One of the common areas for placement of mini-implants in molar distalization is the interdental area.

Mini-implant placement in this area, however, has some disadvantages including:

- Low mechanical stability of the mini-implant due to moderately low thickness of the cortical bone.

- $\quad$ The need to use mini-implants with small diameters is because of inadequate interdental space and there is injury potential to the roots of adjacent teeth.

- The possibility of invasion into the maxillary sinus.

- Inhibition of subsequent tooth movements, etc [58].

Inter-radicular mini-implants have been shown to provide stationary anchorage for various types of orthodontic tooth movement. Mini-implants are frequently used to give skeletal anchorage in the buccal inter-radicular bone. The inter-radicular space is a potentially advantageous region for insertion because there is less potential for complications related to soft tissue irritation, particularly if they are placed through the attached gingiva. While adjacent teeth may restrict mesio-distal tooth movement, buccal interdental mini-implants are extremely beneficial for molar distalization due to their ease of placement and ease of usage during treatment. Three millimetres of distal movement each side can indeed be achieved with a correctly positioned TAD [55].

The highest bucco-lingual thickness was found at the $6 \mathrm{~mm}$ level between the first and second molars. The highest mesio-distal distances, both buccally and palatally were found between the second premolar and the first molar [60].

The advantages of placing a mini-implant buccally are:

- It's ease of placement.

- The presence of adequate cortical bone in posterior maxillary region.
- It is more comfortable to the patient.

- Maintenance of good oral hygiene control.

The disadvantages of placing a mini-implant buccally are:

- Less mesio-distal space is available on the buccal side than on the palatal side. This indicates that, more sites for a safe screw insertion are available on the palatal side than on the buccal side.

- If buccal space is used with accurate implant positioning, distal movement of at least a half cusp width of molar is possible.

- Mini-implants inserted into the inter-radicular space should not interfere with tooth movement when adjacent teeth are moved in an antero-posterior direction. It is likely that extensive molar distal movement is difficult to achieve with buccal inter-radicular mini-implants because the implants would come in contact with the surrounding root during tooth movement.

In the present study, mini-implant with $1.2 \mathrm{~mm}$ x $7 \mathrm{~mm}$ diameter were placed buccally at an angle of $90^{\circ}$ in between second premolar and first molar.

As the molar distalizing force was applied at the level of the bracket, the first molar showed mesio-buccal rotation, uncontrolled distal tipping and intrusion on application of $150 \mathrm{gms}$ (Figure 1-3). On application of 200 gms, the molar showed mesio-buccal rotation, uncontrolled distal tipping, intrusion and extrusion of mesial cusps (Figure 4-6).

\section{Palatal side}

The placement of mini-implants between the buccal roots limits the distal movement of the dentition. Also, this movement causes tipping, especially in the molars.

In MIA-TPA, direct traction from elastic modules spanning from a mini-implant to a TPA helix is used to achieve molar distalization. Mini-implants were inserted into the palate in this study, wherein attached gingiva is not a major concern, in contrast to the buccal side. Moreover, there is more interdental space in the palate relative to the buccal side, and larger mini-implants $(2 \mathrm{~mm}$ in diameter) can be inserted.

The mesio-distal axis of molar movement can be controlled by adjusting the vertical position of mini-implants and/or the direc- 
tion of the line of action relative to furcation. Thus, bodily tooth movement is promoted, rather than tipping movement [55]. Additionally, TPAs are advantageous because to their simplicity and ease of manufacture. The appliance is compact in comparison to other similar devices.

Nalcaci distalized molars via mini-implants with open coil springs by an average $3.95 \pm 1.35 \mathrm{~mm}$. Gelgor distalized molars by an average $3.9 \pm 1.6 \mathrm{~mm}$ via implants supported by TPAs and NiTi open coil springs. Yamada used mini-implants and elastomeric chain or NiTi closed coils for distalization and average molar movement was $2.8 \pm 1.6 \mathrm{~mm}$ [44]. The above study showed that, mean distalization of 4 - $5 \mathrm{~mm}$ was seen with palatal implants on an average duration of 10 - 12 months.

An earlier study, conducted to mechanically evaluate distalization modalities through the application of skeletal anchorage using Finite Element Analysis. Distalization with the palatal plate revealed substantial molar movement and negligible incisor displacement. Placing mini-implants on the buccal side resulted in distally tipped and extruded first molars, whereas labially flared and intruded incisors. Distalization with the palatal plate rather than mini-implants on the buccal side provided bodily molar movement without tipping or extrusion [57]. The above study also inclines towards the opinion that palatal placement of implant is better than buccal.

In this aspect, the palate is an ideal location for mini-implants for maxillary molar distalization because of its adequate cortical bone thickness, which aids in the stability of the mini-implant. In our research, inserting mini-implants on the buccal side resulted in the distal tipping and extrusion of the first molar. The palate on the other hand, presents with thick, dense cortical bone levels, making it one of the most suitable sites for successful MSI placement. The palate, with the exception of the incisive foramen, is a site with a low risk of nerve and blood vessel damage from MSI insertion. Furthermore, the palate is covered with keratinized tissue of ample thickness, which presents an environment that naturally limits tissue irritation and inflammation [59].

The MIA-TPA is an appliance capable of achieving absolute anchoring and successfully driving maxillary first molars distally. Because the distal force is directed apically, extrusion can be avoided. The use of palatal anchorage can also reduce the number of mini- implants required per patient. In most cases, the same MSI can be used for distalization, retraction, or intrusion of teeth, simply by altering the design of the TPA attached [55].

The advantages of palatal implants are:

- They exert force closer to centre of resistance.

- Availability of adequate cortical bone.

- The presence of abundant attached gingiva, more mesiodistal inter dental space, can move the molar by one full cusp width.

The disadvantages of palatal implants are:

- $\quad$ Complicated procedure for implant placement and removal.

- $\quad$ Risk of nerve damage.

In this study, when mini-implant assisted trans palatal arch was used for molar distalization, first molar showed mesio-palatal rotation, distal displacement, intrusion of distal cusps and extrusion of mesial cusps which were the same on application of $150 \mathrm{gms}$ (Figure 7-9) and 200gms (Figure 10-12) of force, but the movements were more with the latter. As the load increases, movement achieved through palatal mini-implant placement was more than buccal mini-implant placement (Graph 3 and 4) (Table 1).

Based on the clinician's requirements and clinical situations, distalization can be achieved by placing the implant buccally or palatally.

\section{Conclusion}

Interradicular mini-implants have been demonstrated to provide fixed anchoring for a variety of orthodontic tooth movements. However, mini-implants inserted into the interradicular space should not interfere with tooth movement when adjacent teeth are moved in an anterior-posterior direction.

Mini-implants placed in the maxillary buccal interradicular space between the second premolar and the first molar at an 90 degree angle were useful for moving maxillary molars distally in non-growing patients: 
- $\quad$ On application of 150 gms, the molar showed mesial out rotation, intrusion with uncontrolled distal tipping.

- $\quad$ On application of 200 gms, the molar showed mesial buccal rotation, intrusion with uncontrolled distal tipping and extrusion of mesial cusps.

- $\quad$ Mini-implants were placed on the palatal interdental area at an angle of 90 degree such that the distalization forces were applied directly to the molar.

- On application of 150 gms, molar showed mesio palatal rotation, distal displacement, intrusion of distal cusps and extrusion of mesial cusps.

- On application of 200 gms of force, molar showed mesio palatal rotation, distal displacement, intrusion of distal cusps and extrusion of mesial cusps, but the movements were increased.

To conclude, both buccal placement and palatal placement of implants have pros and cons such as, buccal placement leads to distalization and unwanted tipping, whereas palatal placement shows more distalization and less tipping, as it is closer to center of resistance. In terms of visibility and ease of placement, buccal implant was easier compared to palatal implant.

\section{Bibliography}

1. Nanda R. "Esthetics and Biomechanics in Orthodontics". $2^{\text {nd }}$ edition. St Louis, MO: Elsevier/Saunders (2015).

2. Trakyali G., et al. "Conscious hypnosis as a method for patient motivation in cervical headgear wear-a pilot study". European Journal of Orthodontics 30.2 (2008): 147-152.

3. Gianelly AA., et al. "Distalization of molars with repelling magnets". Journal of Clinical Orthodontics 22 (1988): 40-44.

4. Gianelly A., et al. "Japanese NiTi coils used to move molars distally". American Journal of Orthodontics and Dentofacial Orthopedics 99.6 (1991): 564-566.

5. Bussick T and McNamara J. "Dentoalveolar and skeletal changes associated with the pendulum appliance". American Journal of Orthodontics and Dentofacial Orthopedics 117.3 (2000): 333-343.

6. Keles A. "Maxillary unilateral molar distalization with sliding mechanics: a preliminary investigation". European Journal of Orthodontics 23.5 (2001): 507-515.
7. Bolla E., et al. "Evaluation of maxillary molar distalization with the distal jet: a comparison with other contemporary methods". The Angle Orthodontist 72 (2002): 481-494.

8. Antonarakis G and Kiliaridis S. "Maxillary Molar Distalization with Noncompliance Intramaxillary Appliances in Class II Malocclusion". The Angle Orthodontist 78.6 (2008): 1133-1140.

9. Fudalej P and Antoszewska J. "Are orthodontic distalizers reinforced with the temporary skeletal anchorage devices effective?" American Journal of Orthodontics and Dentofacial Orthopedics 139.6 (2011): 722-729.

10. Wilmes B and Drescher D. "A miniscrew system with interchangeable abutments". Journal of Clinical Orthodontics 42 (2008): 574-580.

11. Karagkiolidou A., et al. "Survival of palatal miniscrews used for orthodontic appliance anchorage: A retrospective cohort study". American Journal of Orthodontics and Dentofacial Orthopedics 143.6 (2013): 767-772.

12. Baumgaertel S. "Quantitative investigation of palatal bone depth and cortical bone thickness for mini-implant placement in adults". American Journal of Orthodontics and Dentofacial Orthopedics 136.1 (2009): 104-108.

13. Wilmes B., et al. "Insertion Angle Impact on Primary Stability of Orthodontic Mini-Implants". The Angle Orthodontist 78.6 (2008): 1065-1070.

14. Hoste S., et al. "Risk factors and indications of orthodontic temporary anchorage devices: a literature review". Australian Society of Orthodontists 24 (2008): 140-148.

15. Costa A., et al. "Miniscrews as orthodontic anchorage: a preliminary report". The International Journal of Adult Orthodontics and Orthognathic Surgery 13 (1998): 201-209.

16. Choi J., et al. "Bone density measurement in interdental areas with simulated placement of orthodontic miniscrew implants". American Journal of Orthodontics and Dentofacial Orthopedics 136.6 (2009): 766.e1-e12.

17. Park H., et al. "Factors affecting the clinical success of screw implants used as orthodontic anchorage". American Journal of Orthodontics and Dentofacial Orthopedics 130.1 (2006): 18-25.

18. Antoszewska J., et al. "Five-year experience with orthodontic miniscrew implants: A retrospective investigation of factors influencing success rates". American Journal of Orthodontics and Dentofacial Orthopedics 136.2 (2009): 158.e1-e10. 
19. Lee N and Baek S. "Effects of the diameter and shape of orthodontic mini-implants on microdamage to the cortical bone". American Journal of Orthodontics and Dentofacial Orthopedics 138.1 (2010): 8.e1-e8.

20. Park H., et al. "Density of the alveolar and basal bones of the maxilla and the mandible". American Journal of Orthodontics and Dentofacial Orthopedics 133.1 (2008): 30-37.

21. Wilmes B., et al. "Parameters Affecting Primary Stability of Orthodontic Mini-implants". Journal of Orofacial Orthopedics 67.3 (2006): 162-174.

22. Veltri M., et al. "Soft bone primary stability of 3 different mini screws for orthodontic anchorage: A resonance frequency investigation". American Journal of Orthodontics and Dentofacial Orthopedics 135 (2009): 642-648.

23. Liou EJW., et al. "Do mini screws remain stationary under orthodontic forces?" American Journal of Orthodontics and Dentofacial Orthopedics 126 (2004): 42-47.

24. Celenza F and Hochman MN. "Absolute Anchorage in Orthodontics: Direct and Indirect Implant-Assisted Modalities". Journal of Clinical Orthodontics 34 (2000): 397-402.

25. Kanomi R. "Mini-Implant for Orthodontic Anchorage”. Journal of Clinical Orthodontics 31 (1997): 763-767.

26. Serra G., et al. "Sequential bone healing of immediately loaded mini-implants". American Journal of Orthodontics and Dentofacial Orthopedics 134 (2008): 44-52.

27. Pierrisnard L., et al. "Influence of implant length and bicortical anchorage on implant stress distribution". Clinical Implant Dentistry and Related Research 5 (2003): 254-262.

28. Deguchi T., et al. "Quantitative evaluation of cortical bone thickness with computed tomographic scanning for orthodontic implants". American Journal of Orthodontics and Dentofacial Orthopedics 129 (2006): 721.e7-e12.

29. Miyawaki S., et al. "Factors associated with the stability of titanium screws placed in the posterior region for orthodontic anchorage". American Journal of Orthodontics and Dentofacial Orthopedics 124 (2003): 373-378.

30. Roberts WE., et al. "Rigid Implant Anchorage to close mandibular first molar extraction site". Journal of Clinical Orthodontics 28 (1994): 693-704.
31. Kuroda S., et al. "Root proximity is a major factor for screw failure in orthodontic anchorage". American Journal of Orthodontics and Dentofacial Orthopedics 131 (2007): 7.

32. Yettram AL., et al. "Centre of rotation of a maxillary central incisor under orthodontic loading". British Journal of Orthodontics 4 (1977): 23-27.

33. Natali AN., et al. "Experimental-numerical analysis of mini pig's multi-rooted teeth". The Journal of Biomechanics 40 (2007): 1701-1708.

34. Pickard MB., et al. "Effects of mini screw orientation on implant stability and resistance to failure". American Journal of Orthodontics and Dentofacial Orthopedics 137 (2010): 91-99.

35. Oyonarte R., et al. "Peri implant bone response to orthodontic loading: Part 1. A histomorphometric study of the effects of implant surface design". American Journal of Orthodontics and Dentofacial Orthopedics 128 (2005): 173-181.

36. Cacciafesta V., et al. "JCO Round Table: Skeletal Anchorage". Journal of Clinical Orthodontics 63 (2009): 303-317.

37. Halazonetis DJ. "Computer experiments using a two-dimensional model of tooth support". American Journal of Orthodontics and Dentofacial Orthopedics 109 (1996): 598-606.

38. Chen F., et al. "Anchorage effect of various shape palatal osseointegrated implants: a finite element study". The Angle Orthodontist 75 (2005): 378-385.

39. Reynders R., et al. "Mini implants in orthodontics: A systematic review of the literature". American Journal of Orthodontics and Dentofacial Orthopedics 135 (2009): 564-565.

40. Zhao L., et al. "Effect of placement angle on the stability of loaded titanium micro screws: A microcomputed tomographic and biomechanical analysis". American Journal of Orthodontics and Dentofacial Orthopedics 139 (2011): 628-635.

41. Gedrange T., et al. "Three-dimensional analysis of endosseous palatal implants and bones after vertical, horizontal, and diagonal force application". European Journal of Orthodontics 25 (2003): 109-115.

42. Gelgor IE., et al. "Intraosseous Screw-Supported Upper Molar Distalization”. The Angle Orthodontist 74 (2004): 838-850.

43. Sandler J., et al. "Palatal implants are a good alternative to headgear: A randomized trial". American Journal of Orthodontics and Dentofacial Orthopedics 133 (2008): 51-57. 
44. Escobar SA., et al. "Distalization of maxillary molars with the bone-supported pendulum: A clinical study". American Journal of Orthodontics and Dentofacial Orthopedics 131 (2007): 545549.

45. Oberti G., et al. "Maxillary molar distalization with the dualforce distalizer supported by mini-implants:A clinical study". American Journal of Orthodontics and Dentofacial Orthopedics 135.3 (2009): 282-283.

46. Ueno S., et al. "Analysis of force system for upper molar distalization using a trans-palatal arch and mini-implant: A finite element analysis". European Journal of Orthodontics 35.5 (2013): 628-633.

47. Kaya B., et al. "Palatal implant versus zygomatic anchorage for distaliation of maxillary posterior teeth". European Journal of Orthodontics 35.4 (2013): 507-514.

48. Nalcaci R., et al. "A reliable method for evaluating upper molar distalization: Superimposition of three- dimensional digital model". The Korean Journal of Orthodontics 45.2 (2015): 82-88.

49. Mavropoulos A., et al. "Noncompliance unilateral maxillary molar distalization: a three-dimensional tooth movement analysis". The Angle Orthodontist 76 (2006): 382-387.

50. Bondemark L and Kurol J. "Distalization of maxillary first and second molars simultaneously with repelling magnets". European Journal of Orthodontics 14 (1992): 264-272.

51. Brickman CD., et al. "Evaluation of the jones jig appliance for distal molar movement". American Journal of Orthodontics and Dentofacial Orthopedics 118 (2000): 526-534.

52. Ashmore J., et al. "A 3-dimensional analysis of molar movement during headgear treatment". American Journal of Orthodontics and Dentofacial Orthopedics 121.1 (2002): 18-29.

53. Ludwig B., et al. "Application of a new viscoelastic finite element method model and analysis of miniscrew-supported hybrid hyrax treatment". American Journal of Orthodontics and Dentofacial Orthopedics 143 (2013): 426-435.

54. Mavropoulos A., et al. "Efficiency of noncompliance simultaneous first and second upper molar distalization: a three-dimensional tooth movement analysis". The Angle Orthodontist 75 (2005): 532-539.
55. Miresmaeili A., et al. "Three-dimensional analysis of the distal movement of maxillary 1st molars in patients fitted with mini-implants-aided trans-palatal arches". The Korean Journal of Orthodontics Journal 45.5 (2015): 236-244.

56. Duran GS., et al. "Three-dimensional analysis of tooth movements after palatal miniscrew-supported molar distalization". American Journal of Orthodontics and Dentofacial Orthopedics 2150 (2016): 188-197.

57. Yu I., et al. "Comparison of tooth displacement between buccal mini-implants and palatal plate anchorage for molar distalization: a finite element study". European Journal of Orthodontics 36.4 (2011): 394-402.

58. Mohamadi A., et al. "Unilateral Molar Distalization with an Implant-Supported Appliance: A Case Report of a Promising Alternative to Traditional Distalization Techniques". Iranian Journal of Orthodontics 13.1 (2018): 8042.

59. Razavi M. "Applications and Benefits of Fixed Anchorage in the Palate". Orthodontic Perspectives 16.2 (2009): 15-17.

60. Fayed M., et al. "Optimal sites for orthodontic mini-implant placement assessed by cone beam computed tomography". The Angle Orthodontist 280.5 (2010): 939-951.

\section{Volume 5 Issue 10 October 2021 (C) All rights are reserved by Tejaswi Kala., et al.}

